\title{
ANALYSIS OF ALBEDO EFFECTS ON COARSE SUN DIRECTION DETERMINATION ALGORITHMS
}

\author{
Lopes, R. V. F. (1), Silva, A. R. (2), Relloso, J. (3), Absi, G. (4), Jun, Y. (5) \\ (1)INPE, Av. Astronautas 1758, S J Campos, SP, Brazil, roberto.lopes@dss.inpe.br \\ (2) INPE, Av. Astronautas 1758,S J Campos,SP,Brazil, adsilva@dss.inpe.br \\ (3)INVAP, F P Moreno 1089, Bariloche,Argentina, relloso@invap.com.ar \\ (4)INVAP, F P Moreno 1089, Bariloche, Argentina, gabsi@invap.com.ar \\ (5 )CAST, 3 Fuxing Road, Beijing, China,yuanjun2000@sohu.com
}

\begin{abstract}
The purpose of this paper is to analyze the effect of albedo interference on solar sensor measurements in the Survival control mode of Brazilian remote sensing satellite Amazonia-1. Three basic algorithms for coarse Sun vector determination are investigated: the first one selects a subset of sun sensors and ignores albedo effects; the second algorithm also ignores albedo effects but uses the whole set of solar sensors, while the third one takes into account the albedo effects. A possible advantage of increasing the number of solar sensors is investigated by considering an additional set of six solar sensors in cubic configuration. Albedo model follows the 2005 annual average and standard deviation taken from Total Ozone Mapping Spectrometer TOMS Project diffuse reflectivity database, which divides the Earth surface in a set of cells with different reflectance levels. The analysis is mainly based on simulation and includes sensor noise effects. Test scenarios consider a spacecraft in LEO, Sun synchronous orbit at different altitudes, with different local time passes, different albedo reflectance levels, and different spacecraft attitudes. For every test scenario the error in the Sun vector determination is evaluated through one orbit period for different seasons and different GMT. Real data from previous satellites are considered as a model validation source.
\end{abstract}

Keywords: attitude determination, albedo, coarse sun sensor

\section{Introduction}

Coarse Sun vector determination systems are mainly intended to be a source of reliable and robust attitude information to the AOCS in a survival control mode. The Brazilian Multi-Mission Platform MMP has such a system, based on a set of eight cosine-like analog coarse solar sensors spread over the spacecraft corners in an octahedron configuration. Coarse solar sensors are known to be affected by albedo and the subject has been addressed by different authors, as in [1-5].

The purpose of this paper is to analyze the effect of albedo interference on solar sensors measurements in the context of the satellite Amazonia-1. Amazonia-1 is the first Brazilian remote sensing satellite based on MMP. Its AOCS subsystem is currently under development at INVAP, and is being considered as a basis to the AOCS of next Brazilian missions using the MMP. In the survival control mode of Amazonia-1 the AOCS needs to move the spacecraft to a safe attitude condition and maintain it there indefinitely, with the solar panels locked at zero position, looking to the Sun within a coarse tolerance margin.

Three basic algorithms for coarse Sun vector determination are investigated: the first one selects a subset of sun sensors and ignores albedo effects; the second algorithm also ignores albedo effects but uses the whole set of solar sensors, while the third one takes into account the albedo effects. A possible advantage of increasing the number of solar sensors is investigated by considering an additional set of six solar sensors in cubic configuration.

Albedo model follows the annual average and standard deviation taken from Total Ozone Mapping Spectrometer TOMS Project diffuse reflectivity database corresponding to the arbitrarily chosen year 2005. The model divides the Earth surface in a set of cells with different reflectance levels. The resultant albedo in a given solar sensor is a function of several factors: the reflectance level of every Earth surface cell; the daylight condition at every Earth surface cell; the Sun incident angle at 
every Earth surface cell; the area of every Earth surface cell; the satellite visibility condition from every Earth surface cell; the diffuse reflection angle from every Earth surface cell in the satellite direction; the distance from every Earth surface cell to the satellite; the visibility condition of every Earth surface cell from the given solar sensor; and the albedo incident angle from every Earth surface cell in the given solar sensor.

The analysis is mainly based on simulation. Solar sensors are also affected by other error sources like sensor noise, sensor transfer function inaccuracies, aging, sunlight obliteration and reflections on the spacecraft body. In this analysis, sensor noise and albedo are taken as representative of all possible error sources.

Test scenarios consider a spacecraft in LEO, Sun synchronous orbit at different altitudes, with different local time passes, different albedo reflectance levels, and different spacecraft attitudes, since the spacecraft may be tumbling when entering the survival control mode. For every test scenario the error in the Sun vector determination is evaluated through one orbit period for different seasons and different GMT at descending equator crossings.

The analysis is intended to give support to the development of the survival control mode of the AOCS for Amazonia-1 and next satellites based on MMP and may be useful to the development of coarse attitude determination systems affected by albedo. Additionally it provides support to the development of the Electric Ground Support Equipment EGSE of AOCS Subsystem. The average effect of albedo per orbit on solar panels in the survival mode is also a valuable output of the analysis.

\section{The Sun Sensor Set}

\subsection{Sensor Characteristics}

Each CSS is basically a photovoltaic solar cell with hemispheric $(2 \pi$ srd) field of view. Ideally the sensor output is proportional to the cosine of sunlight incidence angle $\theta_{k}$, which is the variable one is interested to observe. From the set of sunlight incidence angles observed by CSSs at different directions the Sun direction is to be determined.

The maximum sensor output $I_{0}$ excluding albedo and possible reflections is achieved with Sun at zero incidence angle, aligned with the CSS boresight. Considering the mean solar irradiance of $1367 \mathrm{~W} / \mathrm{m} 2, I_{0}$ is nominally $16.70 \mathrm{~mA}$.

Actually the solar irradiance decays with the inverse of the square of the distance from Sun to Earth, which is not constant due to the Earth orbit eccentricity. Therefore $I_{0}$ changes correspondently. It also may present variations with time due to solar cell ageing (decay of up to $20 \%$ from BOL to EOL) as well as with temperature and from one CSS to another (uncertainty of $5 \%$ ). Nevertheless, for practical purposes $I_{0}$ is taken as the nominal current for all CSSs during the satellite lifetime. Additionally, part of the sunlight is reflected from the CSS on its cover glass especially at high incidence angles. This causes a non linearity in the sensor transfer function up to $8 \%$ with respect to the cosine law for incidence angles below $70^{\circ}$. The non linearity increases at higher incidence angles. Furthermore, due to the wide sensor field of view the sensor output is affected by albedo from Earth, Moon and parts of spacecraft body.

The sensor output $I_{k}$ is a current proportional to the integral of absorbed radiation power density over the sensor sensitive spectrum band. The CSS are sensitive to a wide spectral bandwidth mainly in the visible to infrared wavelength range.

\subsection{Geometric Configuration}

The set of 8 CSS is distributed over the satellite corners aligned with the normal of the octahedron faces, as shown in Fig. 1. 


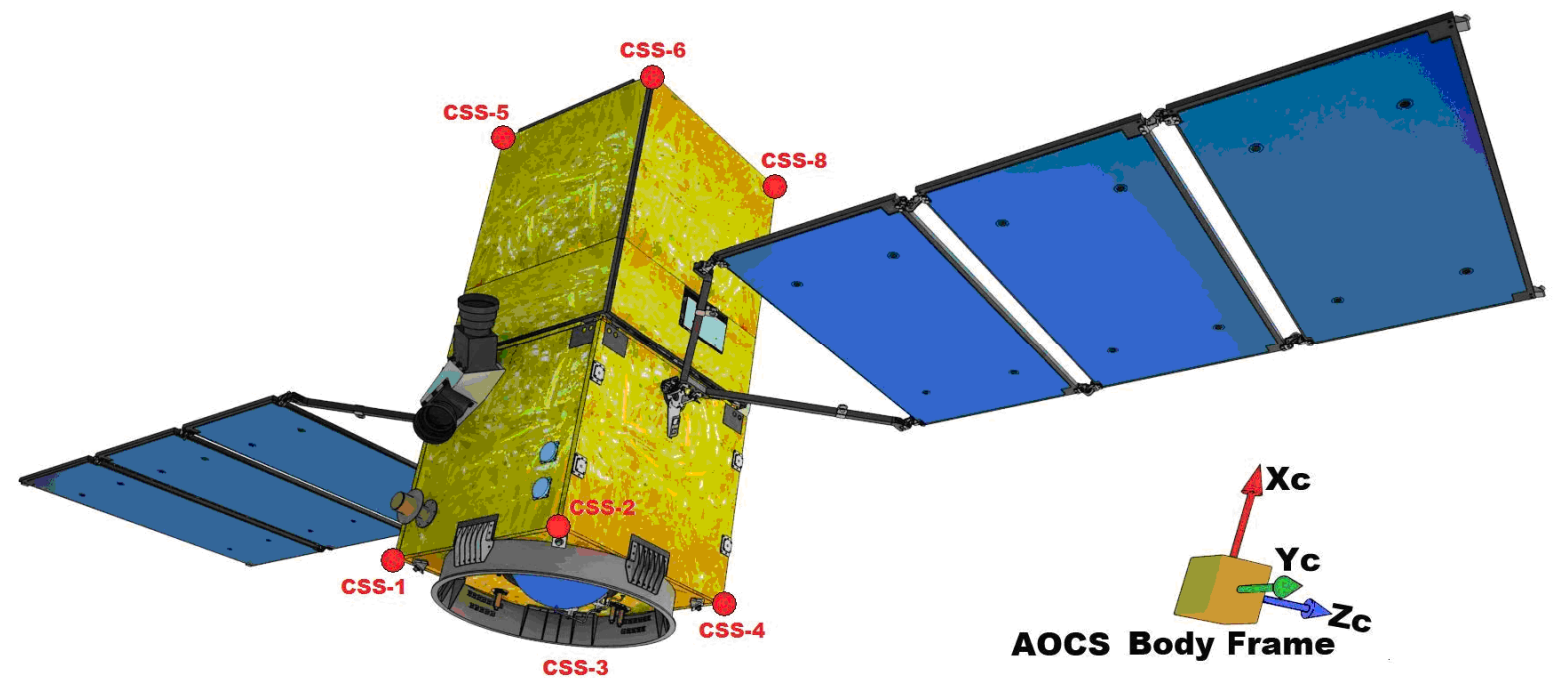

Figure 1 - Distribution of CSS set over the Spacecraft.

The output matrix $\mathrm{H}$ of the CSS set in the AOCS Body Frame (ABF) is given by:

$$
H=\frac{1}{\sqrt{3}}\left[\begin{array}{rrrrrrrr}
-1 & -1 & -1 & -1 & 1 & 1 & 1 & 1 \\
-1 & 1 & -1 & 1 & -1 & 1 & -1 & 1 \\
-1 & -1 & 1 & 1 & -1 & -1 & 1 & 1
\end{array}\right]
$$

\subsection{The Sun Direction Determination Algorithms}

The output of the CSS is a current nearly linear with the incident energy from an hemispheric field of view. Excluding albedo the measurements of k-th CSS can be modeled as:

$$
z_{k}=I_{0} \cos \theta_{k}+v_{k}=I_{0} h_{k}^{\prime} S+v_{k}, \forall k \in[1,8]
$$

Where $\mathrm{S}$ is the Sun direction unit vector, $\theta_{k}$ is the Sun incidence angle at k-th CSS, $I_{0}$ is the sensor nominal output at zero Sun incidence angle, $h_{k}$ is the sensor boresight vector and $v_{k}$ represents the sensor model errors due to several factors already mentioned in section 2.1 besides signal noise.

In matrix notation one has:

$$
Z=I_{0} H^{\prime} S+V
$$

where:

$$
\begin{aligned}
& Z \equiv\left\{\begin{array}{lll}
z_{1} & \cdots & z_{8}
\end{array}\right\}, \\
& V \equiv\left\{\begin{array}{lll}
v_{1} & \cdots & v_{8}
\end{array}\right\} .
\end{aligned}
$$

Algorithm A determines the Sun vector in the AOCS Body Frame in three steps:

i. Evaluate differences between pairs of opposite sensors outputs:

$$
\Delta Z=I_{0} \Delta H^{\prime} S+\Delta V
$$

where:

$$
\Delta=\left[\begin{array}{rrrrrrrr}
1 & 0 & 0 & 0 & 0 & 0 & 0 & -1 \\
0 & 1 & 0 & 0 & 0 & 0 & -1 & 0 \\
0 & 0 & 1 & 0 & 0 & -1 & 0 & 0 \\
0 & 0 & 0 & 1 & -1 & 0 & 0 & 0
\end{array}\right]
$$


ii. Select the three highest differences in absolute value:

$$
\widetilde{\Delta} Z=I_{0} \widetilde{\Delta} H^{\prime} S+\widetilde{\Delta} V
$$

where $\widetilde{\Delta}$ is a matrix with entries correspondent to the three selected rows of $\Delta$. Differences with absolute value smaller than a lower limit $\mu=0.3 I_{0}$ are tagged as not valid to avoid large non linearity errors at high Sun incident angles on the sensors. If the number of valid differences is smaller than two, no solution is computed. If the number of valid differences is equal to two, the highest difference tagged as not valid is exceptionally reconsidered in order to assure observability.

iii. Solve Eq. 8 for Sun unit vector: $S=\frac{\left(\widetilde{\Delta} H^{\prime}\right)^{-1} \widetilde{\Delta} Z}{\left|\left(\widetilde{\Delta} H^{\prime}\right)^{-1} \widetilde{\Delta} Z\right|}$

In this algorithm $\left(\widetilde{\Delta} H^{\prime}\right)$ is always of rank 3 by construction.

Algorithm B eliminates the second step and is based on the pseudo inverse solution of Eq. 6:

$$
S=\frac{\left(H \Delta^{\prime} \Delta H^{\prime}\right)^{-1} H \Delta^{\prime} \Delta Z}{\left|\left(H \Delta^{\prime} \Delta H^{\prime}\right)^{-1} H \Delta^{\prime} \Delta Z\right|}
$$

Both algorithms A and B are mainly independent of $I_{0}$.

Algorithm C considers the pseudo inverse solution of Eq. 6 as a sum of Sun vector $S$ with an equivalent albedo vector $E$ :

$$
S+E=\frac{1}{I_{0}}\left(H \Delta^{\prime} \Delta H^{\prime}\right)^{-1} H \Delta^{\prime} \Delta Z,
$$

The unit vector $S$ is selected from a set of candidates defined by a grid on the unit sphere closer than $40^{\circ}$ from the $S+E$ direction. For each candidate the corresponding albedo vector is evaluated, as well as the residuals of the full set of sensor observations. The solution minimizes the root mean square of the residuals.

\section{Albedo Model}

\subsection{Physical Model}

The albedo simulation follows the Earth reflectance database from Total Ozone Mapping Spectrometer (TOMS) project, taken from [5]. The Earth reflectance is considered diffuse, isotropic, obeying the Lambertian cosine model, with same spectral band of Sun radiation, roughly uniform in the spectral band where the CSS photovoltaic cell is sensitive. Therefore the CSS are considered equally sensitive to both direct sunlight and albedo. Furthermore, solar irradiance absorbed by the Earth and reemitted back to space as a radiation of a black body are neglected, as well as Rayleigh scattering and Stokes reflections phenomena [5].

\subsection{Earth Reflectance Data from TOMS Project}

The TOMS project presents the reflectance index for a grid of elements covering the Earth surface, $D_{i, j}^{\oplus}, i \in[1,180], j \in[1,288]$, with element height $1^{\circ}$ in latitude and element width $1.25^{\circ}$ in longitude. Fig. 2 shows the year average and standard deviation $\sigma$ as well as strong and weak scenarios for year 2005. Iced regions present the highest reflectance and the lowest variation. Variations are mainly due to clouds coverage and seasonal effects. The strong and weak considered scenarios differs from yearly average respectively by $+1 \sigma$ and $-1 \sigma$. 

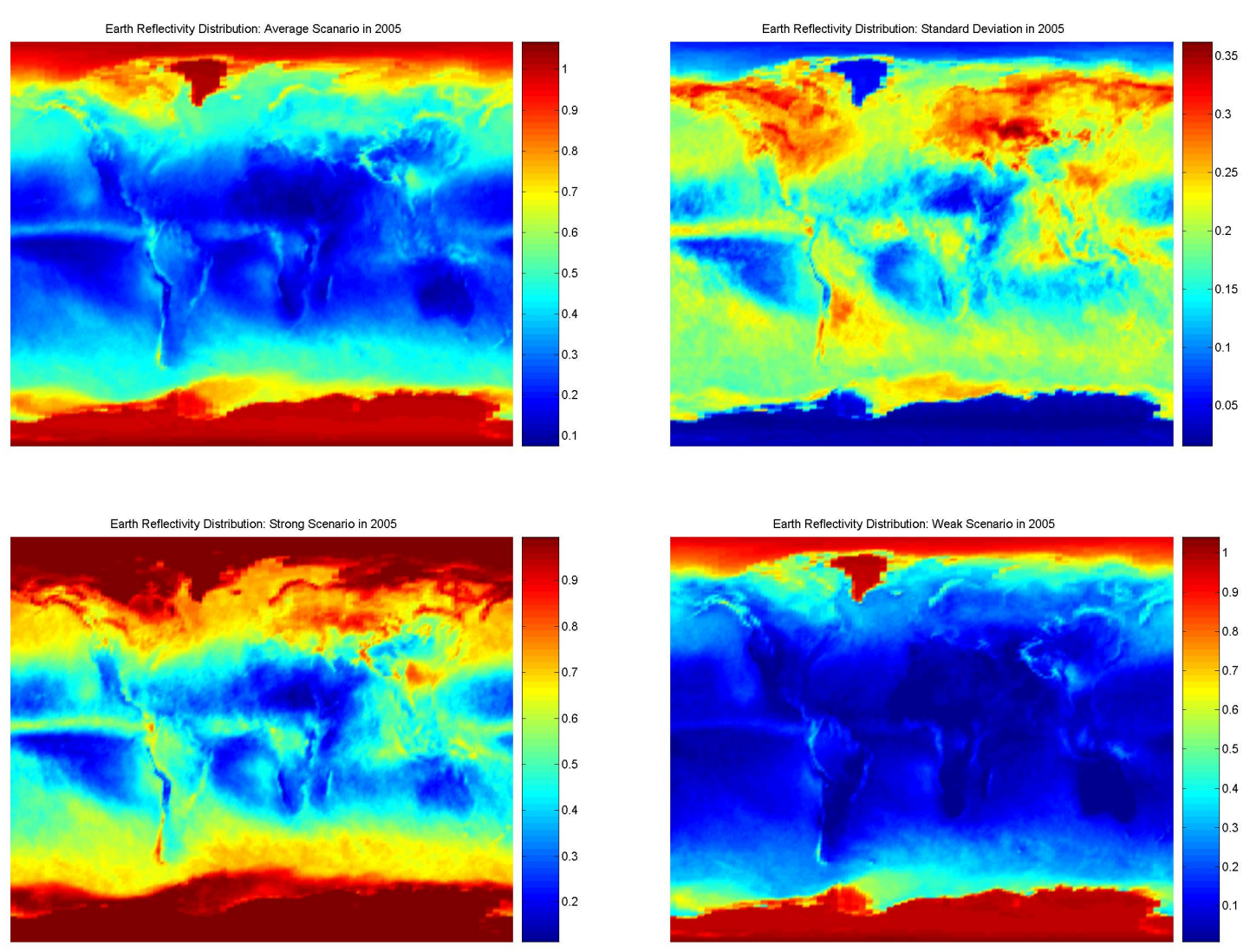

Figure 2 - $\quad$ Earth Reflectance Index for Year 2005: a) Yearly average; b) Standard deviation; c) Strong albedo scenario; d) Weak albedo scenario.

\subsection{Mathematical Model}

The albedo on a given CSS is modeled as the integration of incremental albedo coming from each element $D_{i, j}^{\oplus}$ of the Earth surface grid. Such incremental albedo is a function of four factors: the sunlight incidence angle on $D_{i, j}^{\oplus}$; the satellite elevation angle from $D_{i, j}^{\oplus}$; the satellite distance from $D_{i, j}^{\oplus}$; and the albedo incidence angle from $D_{i, j}^{\oplus}$ on a given sensor. More specifically, the irradiance on the $k$-th CSS due to albedo is given by:

$$
E_{k}=\sum_{i} \sum_{j} \delta E_{i, j, k}
$$

where $\delta E_{i, j, k}$ is the incremental irradiance on $k$-th CSS coming from the element of Earth surface grid $D_{i, j}^{\oplus}$, given by:

$$
\delta E_{i, j, k}=\frac{\Phi_{i, j}}{d_{i, j}^{2}} A_{i, j}^{\oplus} C_{i, j, k}^{\mathrm{CSS}} \cos \theta_{i, j, k}^{\mathrm{CSS}}
$$

where $A_{i, j}^{\oplus}$ is the area of $D_{i, j}^{\oplus}, C_{i, j, k}^{\mathrm{CSS}}$ is the albedo incidence condition on $k$-th CSS from $D_{i, j}^{\oplus}$, $\theta_{i, j, k}^{\mathrm{CSS}}$ is the incidence angle of the incremental albedo at $k$-th CSS from $D_{i, j}^{\oplus}, d_{i, j}$ is the distance 
from $D_{i, j}^{\oplus}$ to the SC, and $\Phi_{i, j}$ is the Earth radiance in the SC direction following the Lambert cosine law for diffuse reflection:

$$
\Phi_{i, j}=\frac{1}{\pi} E^{\mathrm{O}} C_{i, j}^{\mathrm{O}} \cos \theta_{i, j}^{\mathrm{O}} \rho_{i, j}^{\oplus} C_{i, j}^{*} \cos \theta_{i, j}^{*},
$$

and $E^{\mathrm{O}}$ is the solar irradiance, $C_{i, j}^{\mathrm{O}}$ is the sunlight incidence condition on $D_{i, j}^{\oplus}, \theta_{i, j}^{\mathrm{O}}$ is the incidence angle of sunlight on $D_{i, j}^{\oplus}, \rho_{i, j}^{\oplus}$ is the Earth reflectance index at $D_{i, j}^{\oplus}, \theta_{i, j}^{*}$ is the radiance angle from $D_{i, j}^{\oplus}$ in the SC direction, and $C_{i, j}^{*}$ is the spacecraft visibility condition from $D_{i, j}^{\oplus}$, with:

$$
\begin{aligned}
C_{i, j}^{\mathrm{O}} & = \begin{cases}1, & \forall \theta_{i, j}^{\mathrm{O}} \mid \cos \theta_{i, j}^{\mathrm{O}}>0 \\
0, & \text { otherwise }\end{cases} \\
C_{i, j}^{*} & = \begin{cases}1, & \forall \theta_{i, j}^{*} \mid \cos \theta_{i, j}^{*}>0 \\
0, & \text { otherwise }\end{cases} \\
C_{i, j, k}^{\mathrm{CSS}} & = \begin{cases}1, & \forall \theta_{i, j, k}^{\mathrm{CSS}} \mid \cos \theta_{i, j, k}^{\mathrm{CSS}}>0 \\
0, & \text { otherwise }\end{cases}
\end{aligned}
$$

Figure 3 illustrates the effect of $\rho_{i, j}, C_{i, j}^{\mathrm{O}}, \theta_{i, j}^{\mathrm{O}}, C_{i, j}^{*}$ and $\theta_{i, j}^{*}$ on $\Phi_{i, j}$. Figure 3 a shows the Earth irradiance at an arbitrary GMT and season and Figure $3 \mathrm{~b}$ shows the correspondent Earth albedo as seen from a spacecraft at $750 \mathrm{~km}$ of altitude on the average albedo scenario. The effect of GMT in two different seasons and under moderate Earth albedo level is seen in Fig. 4, where GMT changes in the horizontal plane and the orbit path in the vertical plane.

The effect of albedo on a given CSS is an additional current $\delta I_{k}$ given by:

$$
\delta I_{k}=I_{0} \frac{E_{k}}{E^{\mathrm{O}}} .
$$

Again, since $E_{k}$ is proportional to $E^{\mathrm{O}}$, the final effect on the Sun direction is independent of $I_{0}$ and $E^{\mathrm{O}}$ values.
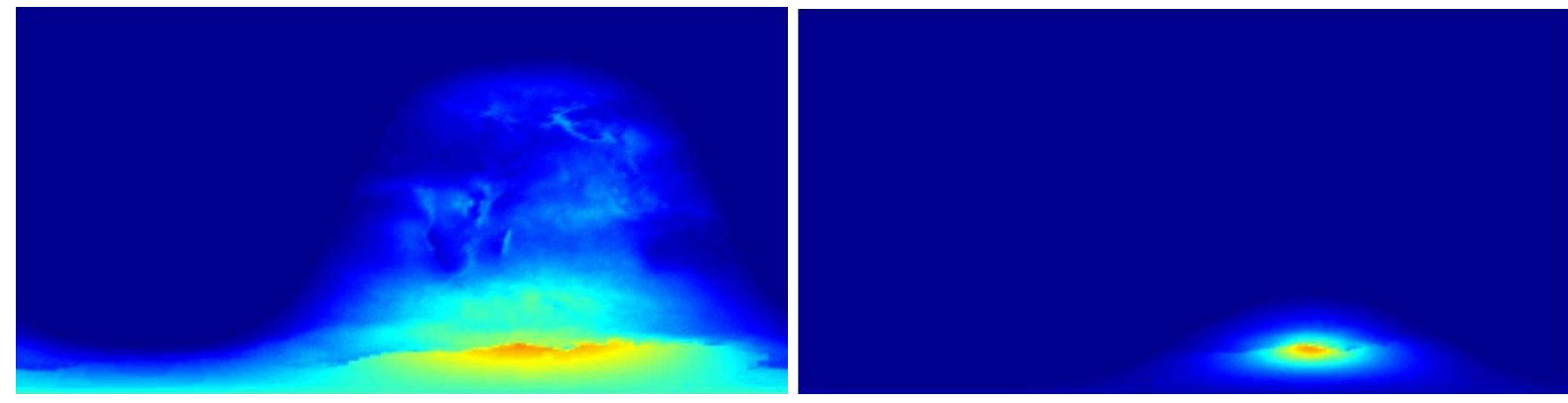

Figure 3 - $\quad$ Example of: a) Earth Irradiance; b) Earth Radiance as seen by the spacecraft.

\section{Test results}

\subsection{Test Scenarios}

Each test is carried out considering a reference test scenario described below, spacecraft at Earth pointing attitude exposed to albedo with moderate level. The first algorithm is also tested against different test scenarios: Sun pointing and off pointed attitudes and other albedo levels. 

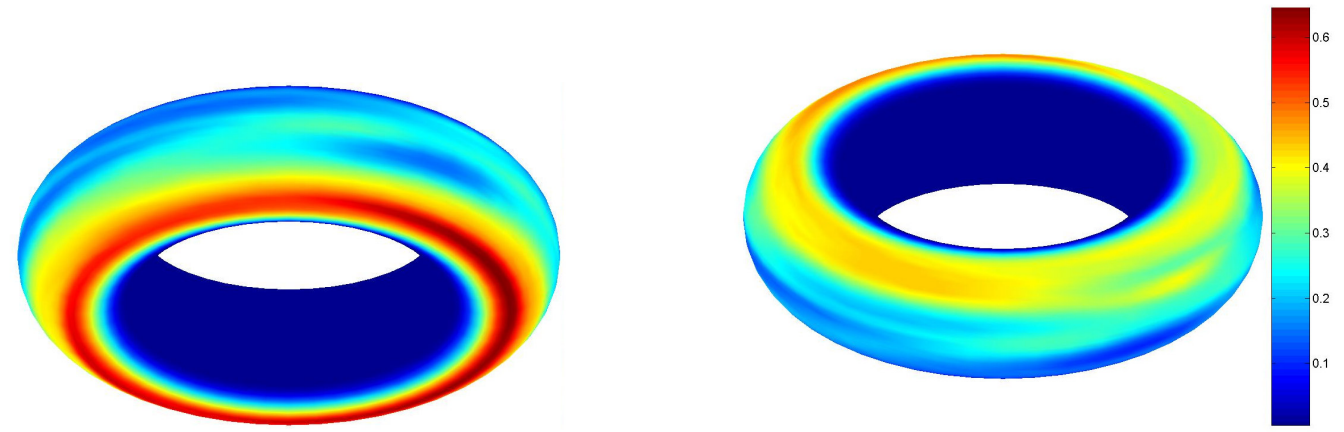

Figure 4 - $\quad$ Albedo Irradiance at spacecraft throughout the orbit and GMT at different seasons:

a) January; b) July.

Orbit Parameters:

Equatorial altitude: $753 \mathrm{~km}$

Inclination: $98.405^{\circ}$ (sun synchronous orbit condition)

Eccentricity: 0.001111 (frozen orbit)

Longitude of ascending node: corresponding to descending equator crossings at 10:30

Argument of perigee: $90^{\circ}$ (frozen orbit)

Mean anomaly: from $0^{\circ}$ to $360^{\circ}$, at steps of $5^{\circ}$

Epoch, Time, Attitude and Albedo Level:

Epoch: from beginning to end of year, steps of $\frac{1}{2}$ month;

GMT at descending equator crossings: from 0 to 24 hours, step of $\frac{1}{2}$ hour;

Attitude: Earth oriented; Sun oriented (nominal and off pointed by $30^{\circ}$ in pitch and yaw);

Albedo level: moderate, strong and weak.

The effect of decreasing the orbit altitude by $100 \mathrm{~km}$ in the reference test scenario on algorithms A and $\mathrm{B}$ is investigated, while the effect of adding 6 extra CSS to the sensor set in the reference test scenario is investigated for algorithms $\mathrm{B}$ and $\mathrm{C}$ only, because they are the most potentially able to be benefited by observation redundancy.

\subsection{Albedo Effect on Each CSS}

Figure 5 shows the Sun incidence angle in each CSS during one orbit along the whole year with the spacecraft at Earth pointing attitude. In this mode CSS 3 and 7, and especially CSS 1 and 5 look more directly to Sun because they are placed on lateral SC panel facing East from where sunlight comes at Amazonia-1 descending passes, while CSS 2, 4, 6 and 8 offer only lateral views.

There are always four CSS seeing the Sun and every CSS sees the Sun in different periods, but only CSS 3, 4, 7 and 8 are more affected by albedo. This may be seen in Fig. 6 where albedo variations along the orbit and during the whole year are shown for each individual CSS.

The magnitudes in Figure 6 represent the average over all GMTs of irradiance at each CSS due to Earth albedo as a fraction of maximum direct solar irradiance. Albedo effect on CSSs peaks over South Pole during the summer on South hemisphere.

Analogously, Figs. 7 and 8 show Sun incidence angle and albedo on each CSS when the spacecraft is oriented to the Sun with off pointing of $30^{\circ}$ in pitch and yaw. In this case CSS 1 to 4 always see the Sun because they are placed on the panel that is oriented in the Sun direction, while CSS 5 to 8 never see the Sun and are the most affected by albedo. The albedo irradiance on CSS 5 and 6 peaks over South Pole in summer, while CSS 7 and 8 are mostly influenced over North Pole in summer.

\subsection{Albedo effect on Sun direction}

Figure 9 shows the relatively weak variation on the accuracy pattern of major, middle and minor sensors with GMT as well as on the respective Sun direction under moderate Earth albedo. Empty 
cells refer to night and other orbit parts where the condition stated in the second step of Sun direction determination algorithm fails to be true and consequently no solution can be computed.
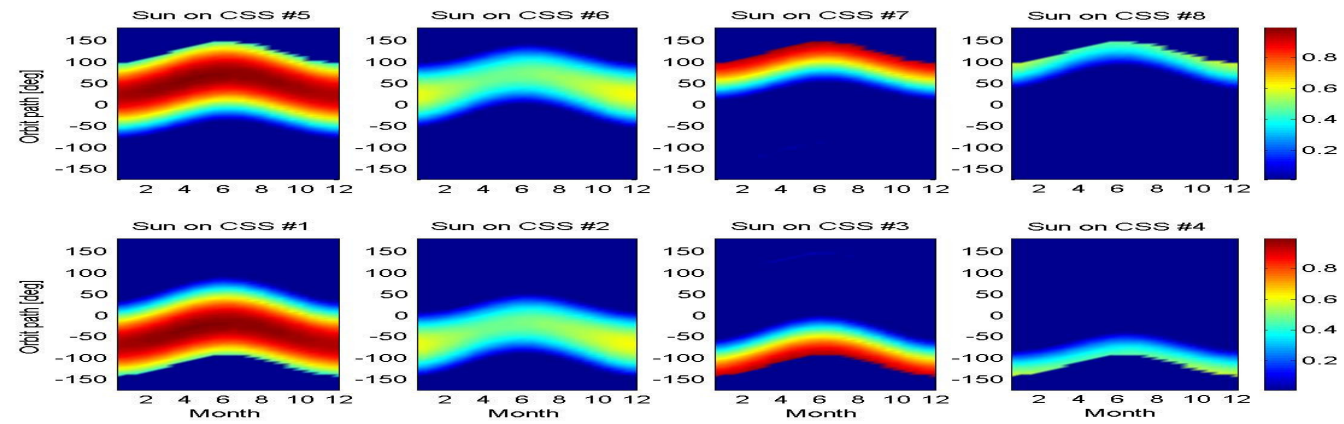

Figure 5 - Sun Incidence on Each CSS: Erth pointing
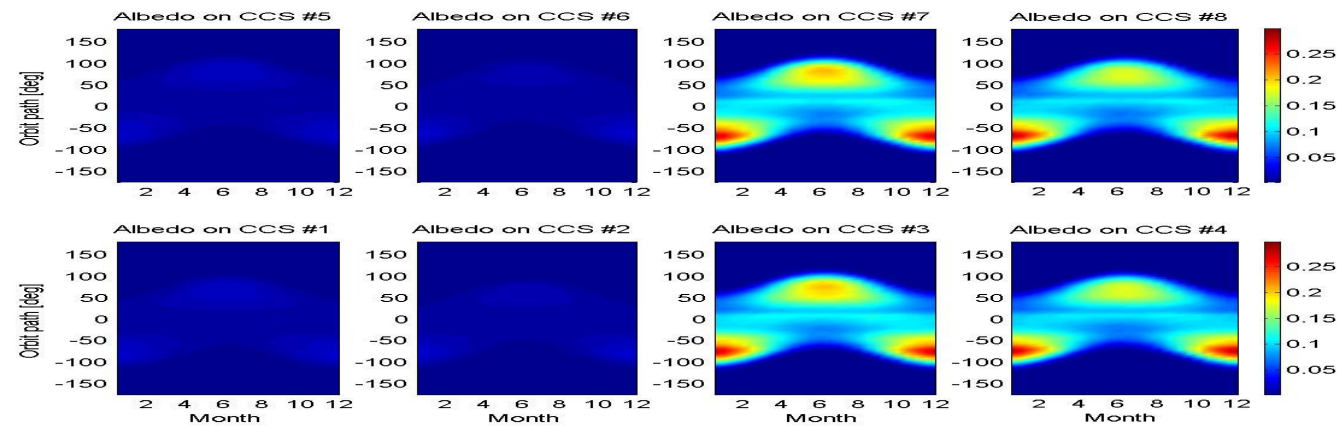

Figure 6 - Albedo average effect on every sensor during one orbit along the year: Earth pointing
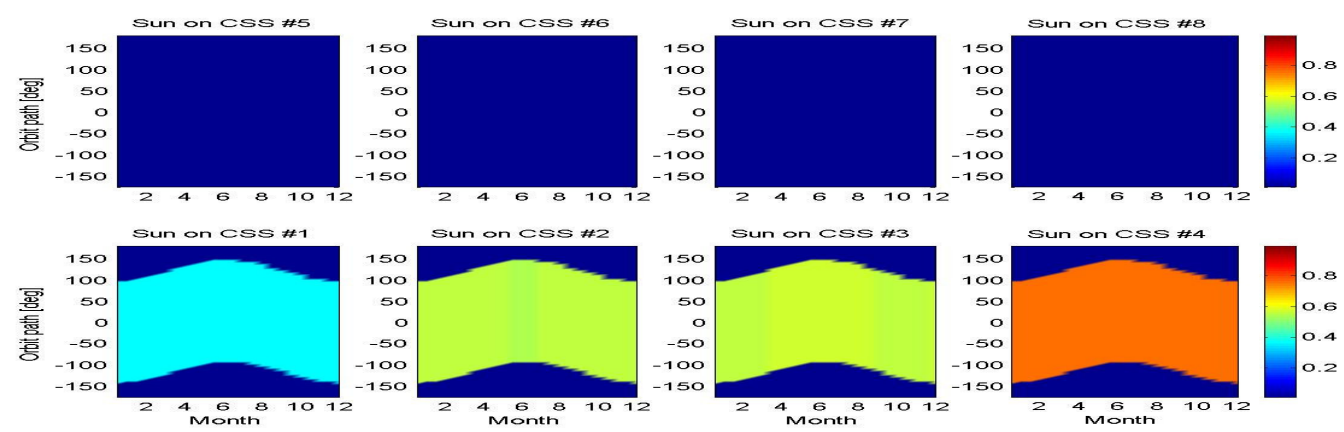

Figure 7 - Sun Incidence on Each CSS: Sun oriented attitude, off- pointed in pitch and yaw.
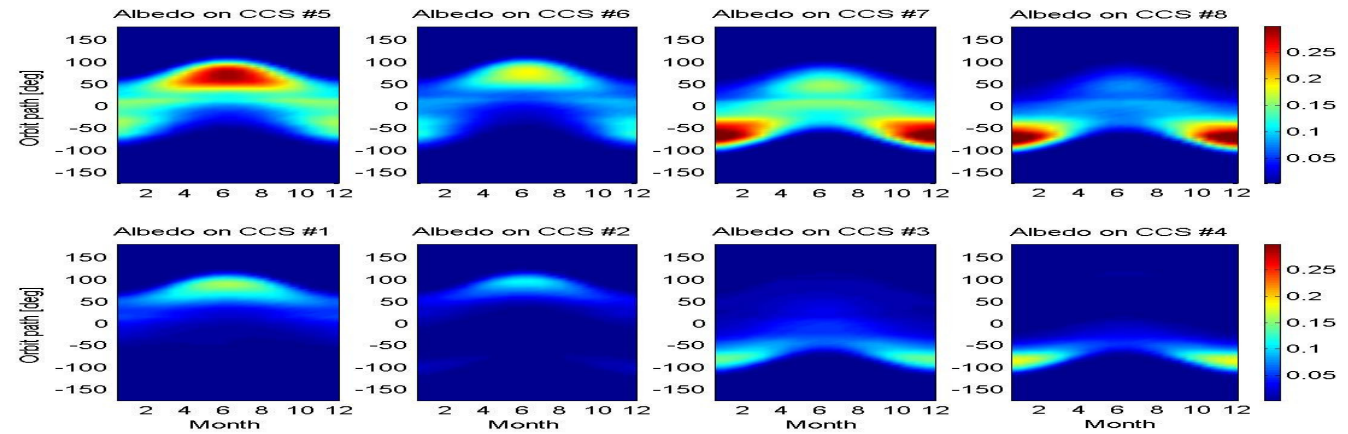

Figure 8 - Albedo average effect on every sensor during one orbit along the year: Sun oriented attitude, off- pointed in pitch and yaw. 

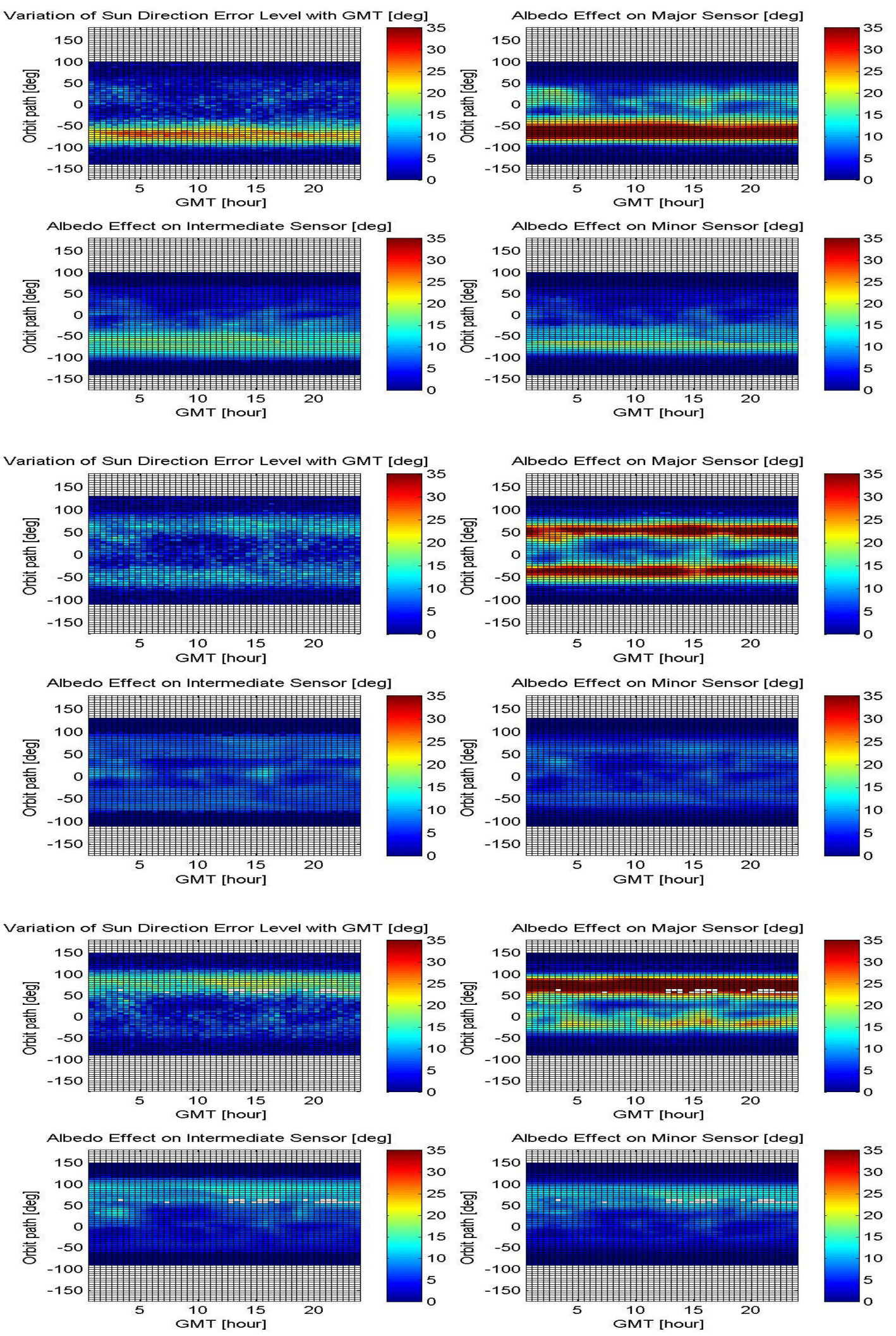

Figure 9 - Effect of GMT on accuracy of: a) Sun direction; b) major; c) middle; d) minor sensors under moderate albedo at different year seasons: A) January; B) April; C) July.

Figure 10 shows the seasonal variation of the accuracy pattern averaged over all GMT on major, middle and minor sensors as well as on the respective Sun direction under moderate Earth albedo 
level. The effect of albedo is highest on the major sensor due to the difference between a CSS looking straight to the Sun and its opposite CSS, which looks straight to Earth. This effect peaks on summer in both hemispheres at polar latitudes. Magnitudes on Fig. 10 refer to average values over all GMT, while Figure 11 shows the accuracy performance under the same moderate Earth albedo levels at worst case GMT along the year.
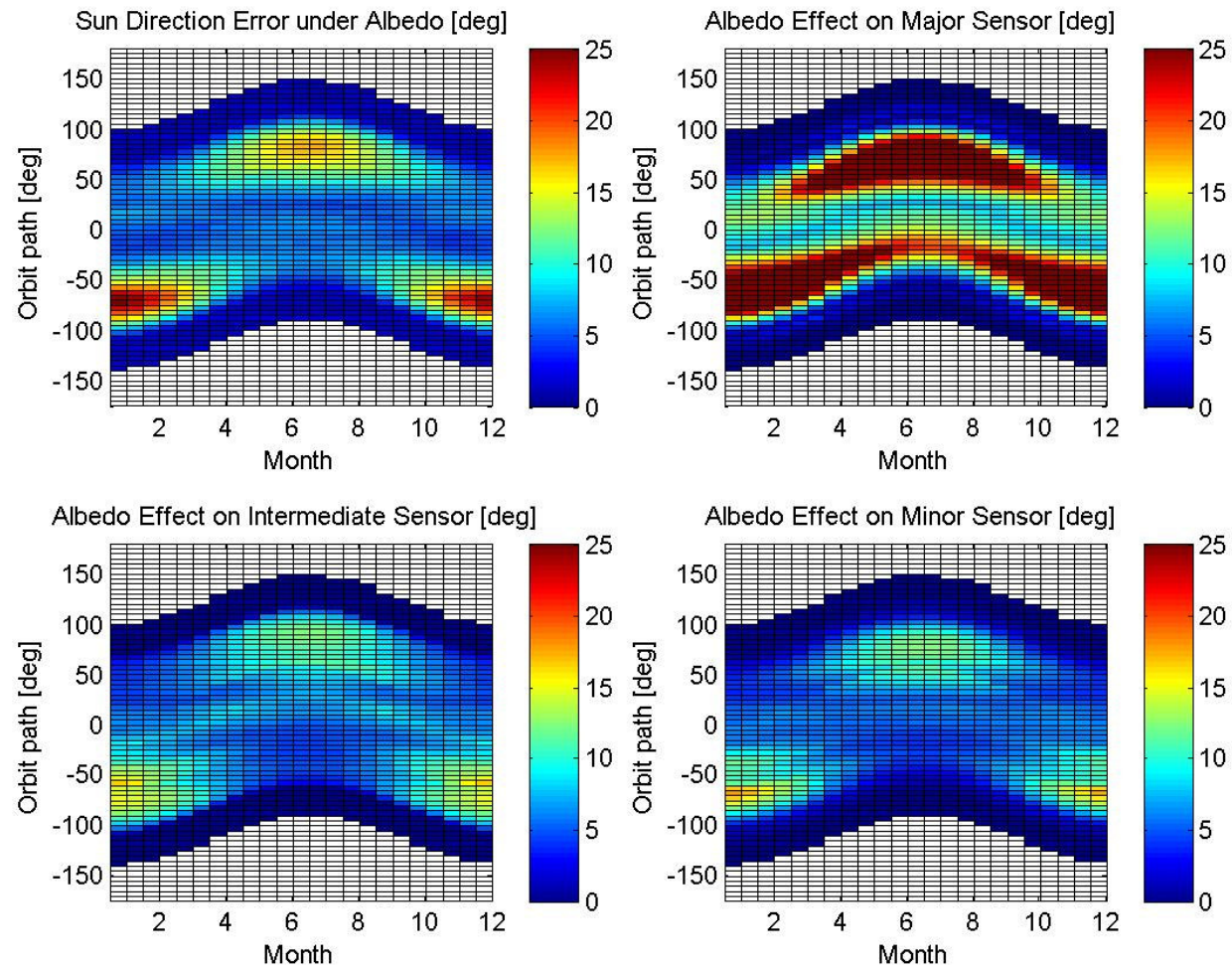

Figure 10 - Seasonal effect on average accuracy of: a) Sun direction; b) major sensor; c) middle sensor; d) minor sensor under moderate Earth albedo level and Earth pointing.

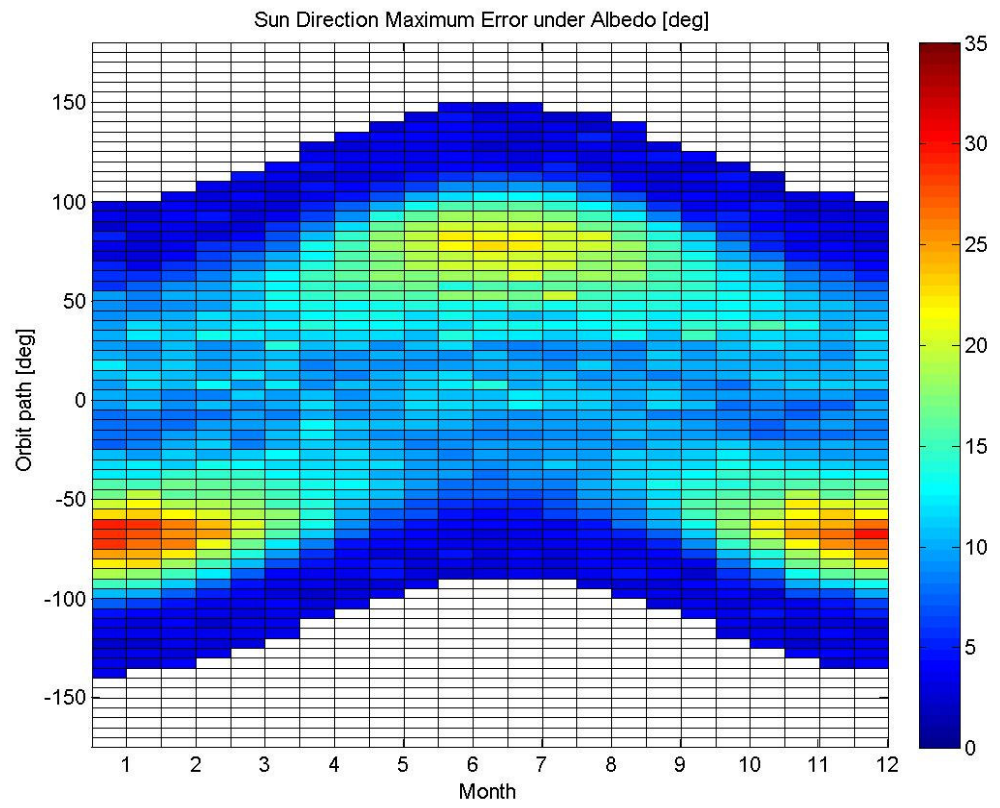

Figure 11 - Seasonal effect on accuracy of Sun direction at worst case GMT under moderate Earth albedo level and Earth pointing. 


\subsection{Additional Test with Different Test Scenarios and Algorithms}

Analogously, Figs. 12 and 13 show the effect of albedo for a Sun oriented attitude, with off pointing of $30^{\circ}$ on pitch and yaw. The strongest albedo effect is now at the minor CSS, but the effect on Sun direction is quite similar to the Earth oriented case.
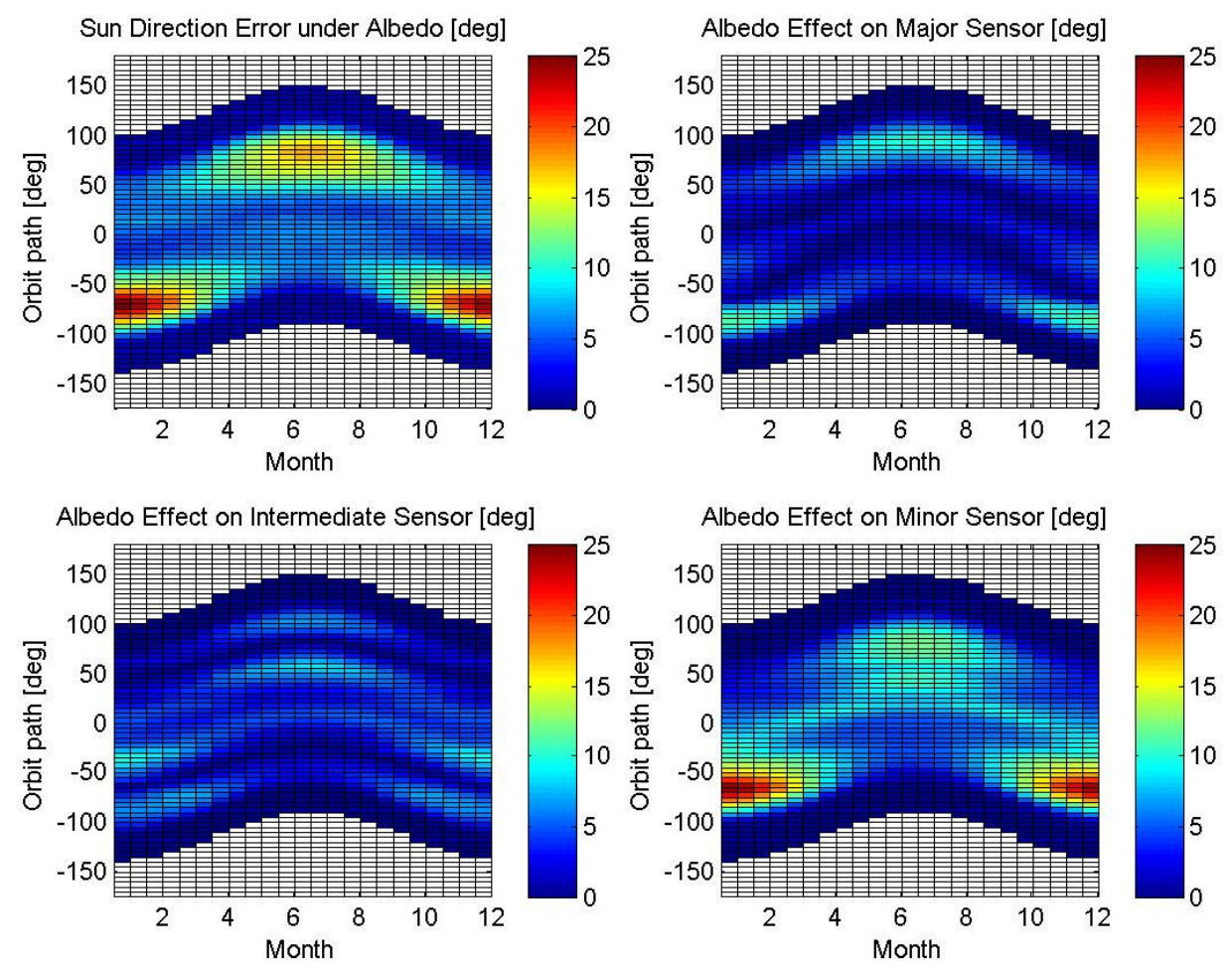

Figure 12 - Seasonal effect on average accuracy of: a) Sun direction; b) major sensor; c) middle sensor; d) minor sensor under moderate Earth albedo level and Sun pointing.

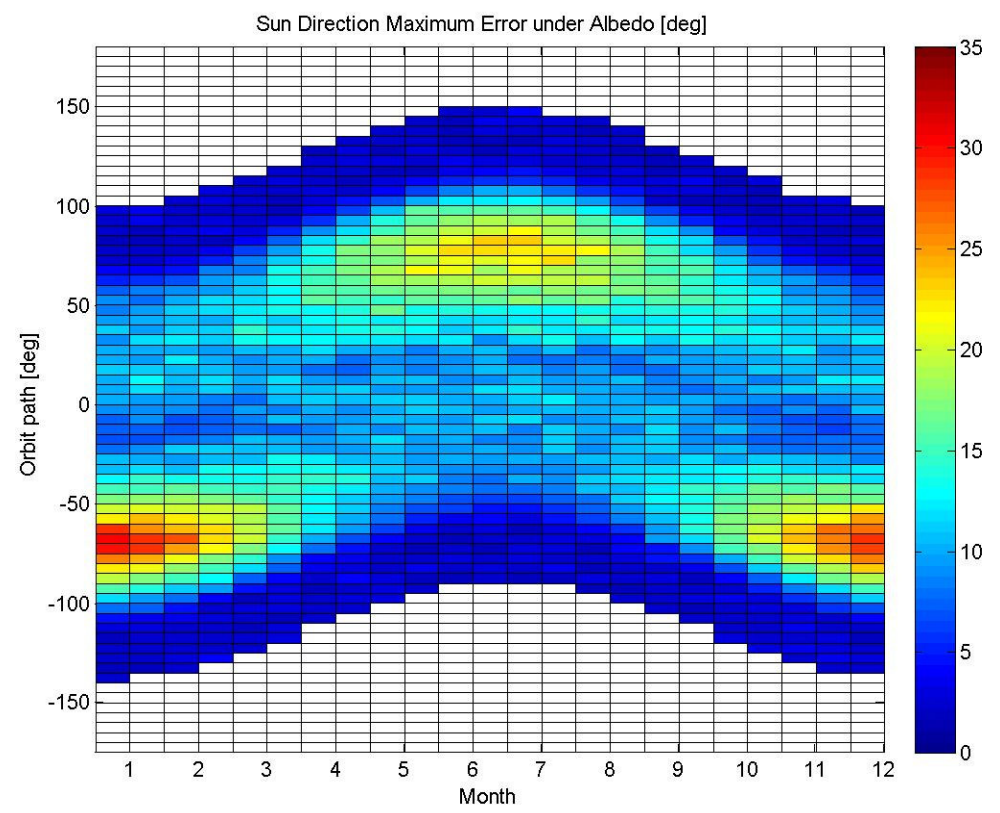

Figure 13 - Seasonal effect on accuracy of Sun direction at worst case GMT under moderate Earth albedo level and Sun pointing. 
Table 1 summarizes the results of test cases with algorithm A. Table 2 summarizes the results of test cases with different orbit altitudes, number of sensors and algorithms, while Tab. 3 summarizes the results of test cases with the reference test scenario but with different local times.

Table 1 - Summary of numerical results: Algorithm A, orbit altitude $753 \mathrm{~km}$

\begin{tabular}{|c|c|c|c|c|c|c|c|c|}
\hline \multirow{4}{*}{} & \multicolumn{3}{|c|}{ Sun Off Pointing } & \multicolumn{4}{c|}{ CSS } & \multicolumn{2}{c|}{ Sun direction } \\
\cline { 2 - 9 } Albedo & $\begin{array}{c}\text { Roll } \\
{[\mathrm{deg}]}\end{array}$ & $\begin{array}{c}\text { Pitch } \\
{[\mathrm{deg}]}\end{array}$ & $\begin{array}{c}\text { Yaw } \\
{[\mathrm{deg}]}\end{array}$ & $\begin{array}{c}\text { Major } \\
{[\mathrm{deg}]}\end{array}$ & $\begin{array}{c}\text { Middle } \\
{[\mathrm{deg}]}\end{array}$ & $\begin{array}{c}\text { Minor } \\
{[\mathrm{deg}]}\end{array}$ & $\begin{array}{c}\text { Average } \\
{[\mathrm{deg}]}\end{array}$ & $\begin{array}{c}\text { Worst Case } \\
{[\mathrm{deg}]}\end{array}$ \\
\hline \multirow{2}{*}{$\begin{array}{c}\text { Low } \\
\text { Level }\end{array}$} & 0 & 0 & 0 & 1.7 & 1.5 & 2.9 & 3.8 & 29.8 \\
\cline { 2 - 10 } & - & - & Nadir & 6.7 & 3.0 & 2.5 & 4.1 & 28.2 \\
\hline \multirow{3}{*}{$\begin{array}{c}\text { Moderat } \\
\text { e Level }\end{array}$} & 0 & 0 & 0 & 2.7 & 2.9 & 5.5 & 6.5 & 30.1 \\
\cline { 2 - 10 } & 0 & 0 & 30 & 4.2 & 4.7 & 6.3 & 6.7 & 30.4 \\
\cline { 2 - 10 } & 0 & 30 & 30 & 6.3 & 3.1 & 6.2 & 6.8 & 31.0 \\
\cline { 2 - 9 } & - & - & Nadir & 12.1 & 5.6 & 4.9 & 6.7 & 30.1 \\
\hline \multirow{2}{*}{$\begin{array}{c}\text { High } \\
\text { Level }\end{array}$} & 0 & 0 & 0 & 3.8 & 4.3 & 8.1 & 9.8 & 34.5 \\
\cline { 2 - 9 } & - & - & Nadir & 17.3 & 8.0 & 7.2 & 9.9 & 34.0 \\
\hline
\end{tabular}

Table 2 - Summary of numerical results: effect of orbit altitude, number of sensors and algorithms

\begin{tabular}{|c|c|c|c|c|c|c|}
\hline \multicolumn{4}{|c|}{ Test Scenario } & \multirow{2}{*}{} & \multicolumn{2}{c|}{ Sun Direction Accuracy } \\
\cline { 1 - 3 } $\begin{array}{c}\text { Albedo } \\
\text { level }\end{array}$ & Attitude & $\begin{array}{c}\text { Orbit } \\
\text { Altitude } \\
{[\mathrm{km}]}\end{array}$ & $\begin{array}{c}\text { Number of } \\
\text { Sensors }\end{array}$ & Algorithm & $\begin{array}{c}\text { Average } \\
{[\mathrm{deg}]}\end{array}$ & Worst Case [deg] \\
\hline Moderate & Earth oriented & 753 & 8 & A & 6.7 & 30.1 \\
\hline Moderate & Earth oriented & 753 & 8 & B & 6.5 & 28.8 \\
\hline Moderate & Earth oriented & 753 & 8 & C & 1.6 & 32.5 \\
\hline Moderate & Earth oriented & 653 & 8 & A & 7.0 & 31.1 \\
\hline Moderate & Earth oriented & 653 & 8 & B & 6.8 & 31.0 \\
\hline Moderate & Earth oriented & 753 & 12 & B & 6.4 & 29.3 \\
\hline Moderate & Earth oriented & 753 & 12 & C & 1.4 & 7.1 \\
\hline
\end{tabular}


Table 3 - Summary of numerical results: effect of local time at descending equator crossings

\begin{tabular}{|c|c|c|c|c|c|c|}
\hline \multirow{3}{*}{ Local Time } & \multirow{3}{*}{ Algorithm } & \multicolumn{5}{|c|}{ Performance [deg] } \\
\hline & & \multicolumn{3}{|c|}{ CSS } & \multicolumn{2}{|c|}{ Sun direction } \\
\hline & & $\begin{array}{c}\text { Majo } \\
\mathrm{r}\end{array}$ & $\begin{array}{l}\text { Middl } \\
\mathrm{e}\end{array}$ & $\underset{r}{\text { Mino }}$ & Average & Worst Case \\
\hline \multirow{2}{*}{ Sunset } & A & \multirow{2}{*}{1.8} & \multirow{2}{*}{2.1} & \multirow{2}{*}{1.8} & 3.4 & 27.9 \\
\hline & $\mathrm{C}$ & & & & 1.7 & 21.7 \\
\hline \multirow{2}{*}{$10: 30$} & A & \multirow{2}{*}{12.1} & \multirow{2}{*}{5.6} & \multirow{2}{*}{4.9} & 6.7 & 30.1 \\
\hline & $\mathrm{C}$ & & & & 1.6 & 32.5 \\
\hline \multirow{2}{*}{ Noon } & A & \multirow{2}{*}{7.5} & \multirow{2}{*}{7.1} & \multirow{2}{*}{5.2} & 6.4 & 29.5 \\
\hline & $\mathrm{C}$ & & & & 1.7 & 7.2 \\
\hline
\end{tabular}

\section{Analysis of Test Results}

The Earth albedo affects the CSS measurements in different ways that varies along the orbit, GMT at descending equator crossings, year season and level of Earth albedo. As observed from all simulation results, worse conditions are over the South and North Poles and during their respective summer season, when the Sun direction error becomes remarkably higher than its average value. The lack of observability phenomenon eventually arose mainly under strong albedo scenario in the Earth oriented attitude and at latitudes right below the Polar circles during their summer seasons. Sometimes in such conditions the coarse solar sensors set failed to offer at least two valid difference observations during relatively short periods. The phenomenon was not observed in Sun oriented attitude and therefore presents little concern regarding the feasibility of the survival control mode. Besides the observability issue, no other meaningful difference was observed in the algorithms performance with respect to the spacecraft orientation.

Algorithm A presented Sun direction errors ranging from $3^{\circ}$ to $10^{\circ}$ in average and up to $35^{\circ}$ in the worst case for an orbit altitude about $750 \mathrm{~km}$. Decreasing the orbit altitude by $100 \mathrm{~km}$ slightly increased the albedo effect on Sun direction accuracy by less than $5 \%$. Expectedly, orbits with local time at equator crossings closer to noon are more affected by albedo than those closer to sunset. In general the error magnitudes were more sensitive to orbital latitude and year season than to GMT at equator crossings.

Despite being manageable by the attitude control system in a survival control mode, those error magnitudes could be mitigated by an effort on algorithm design and sensor set configuration.

Processing the full set of observations from the coarse sun sensors as provided by algorithm B presented little improvement on Sun direction accuracy even with the six additional sensors. Nevertheless, the albedo compensation method implemented by algorithm $\mathrm{C}$ presented a remarkable improvement especially on the average accuracy of Sun direction. The maximum error could also decreases significantly by adding six solar sensors to the original set.

The observed error average range of algorithm A agrees with some reported data [2] and is mainly consistent with the previous experience of the authors from real data. The worst case analysis refers to a set of simultaneous conditions seldom observed in practice. The benefits from algorithm $\mathrm{C}$ foreseen by the current analysis recommend it to further investigations.

The current analysis was based on the albedo model from TOMS project that considers observed Earth irradiance index for each latitude and longitude. Additionally, a simplified model was implemented and compared with the first one, which considers the resultant irradiance on each CSS due to Earth albedo as coming from a single point energy source like a secondary, virtual Sun. 
Comparing with the full model, this simplified model presented results with a discrepancy in the order of magnitude of $20 \%$ to $30 \%$ in average and less than $40 \%$ in the worst case. Considering the difficulty in predicting the daily variation of Earth albedo and the observed standard deviation about average levels such discrepancy may be considered acceptable for testing purposes to the AOCS software in the scope of the Electrical Ground Support Equipment to the subsystem.

\section{Final Comments and Conclusions}

A comprehensive analysis of Earth albedo effects on coarse sun sensor measurements under several test scenarios was carried out from numerical simulation based on TOMS project albedo database. From the point of view of Attitude Control Subsystem in survival control mode the main effect refers to the Sun direction determination accuracy. Using a simple algorithm that selects three pairs of opposite coarse sun sensors with highest observed differences the Sun direction presented average error magnitudes ranging from $3^{\circ}$ under weak albedo scenario to $10^{\circ}$ under strong albedo scenario and up to $35^{\circ}$ in the worst case for an orbit altitude about $750 \mathrm{~km}$. The performance became about $5 \%$ worse for an orbit $100 \mathrm{~km}$ closer to Earth. Worst cases happened over both Poles during summer and are less dependent on albedo scenario. The spacecraft attitude presented little effect on the accuracy and so did the GMT at equator crossings. Sun synchronous orbits with early passes are less sensitive to albedo than those with local time closer to noon. Increasing the number of processed observations presented minor improvements to the accuracy.

A proposed albedo compensation technique based on the determination of an equivalent albedo vector improved the average accuracy of Sun direction by a factor up to four. Similar improvements referring to worst cases were possible with six additional sensors. From a practical point of view the technique would require further investigations in order to overcome two main drawbacks to its implementation as part of a survival control mode. First, the algorithm requires accurate and regularly updated knowledge of the sensitivity of each solar sensor during the spacecraft lifetime. This could be provided by a periodic on board calibration effort. Second, the number of required numeric evaluations is conflicting with the high reliability requirements of a survival control mode.

A simplified model based on an equivalent albedo vector presented a discrepancy with respect to the full model in the order of magnitude of $20 \%$ to $30 \%$ in average and less than $40 \%$ in the worst case, and could be suitable to software test purposes of the attitude control subsystem in survival control mode.

Finally, the amount of albedo on the Solar Array Generator compensated energy losses due to a Sun pointing error under inaccurate Sun direction knowledge in the survival control mode.

\section{References}

[1] Fisher, H. L., Musser, K. L., and Shuster, M. D., _Coarse attitude determination from Earth albedo measurements, IEEE Transactions on Aerospace and Electronic Systems, 1993.

[2] Brasoveanu, D. and Sedlak, J., "Analysis of earth albedo effect on sun sensor measurements based on theoretical model and mission experience." (AAS 98-338) AAS/GSFC 13th International Symposium on Space Flight Dynamics, Vol. 1, May 1998, pp. 485_497.

[3] Appel, P. "Attitude estimation from magnetometer and earth-albedo-corrected coarse sun sensor measurements", Acta Astronautica 56 (2005) 115 - 126.

[4] Bhanderi, D., Bak, T., Modeling Earth Albedo for Satellites in Earth Orbit, [AIAA-2005-6465] AIAA Guidance, Navigation, and Control Proceedings, 15-18 August, 2005. Session 117-GNC-75.

[5] D. Bhanderi, Spacecraft attitude determination with Earth albedo corrected sun sensor measurements. Ph.D. Thesis, Aalborg University, Denmark, August 2005. 